\title{
A Cost-Effectiveness-Assessing Model of Vaccination for Varicella and Zoster
}

\author{
M. Comba ${ }^{1}$, S. Martorano-Raimundo ${ }^{2}$, E. Venturino ${ }^{1 *}$ \\ ${ }^{1}$ Dipartimento di Matematica "Giuseppe Peano", via Carlo Alberto 10 \\ Università di Torino, 10123 Torino, Italia \\ ${ }^{2}$ Faculdade de Medicina, Universidade de São Paulo, Instituto Oscar Freire \\ CEP: 05405-000, Rua Teodoro Sampaio, 115, São Paulo, S.P., Brazil
}

\begin{abstract}
A decision analytical model is presented and analysed to assess the effectiveness and cost-effectiveness of routine vaccination against varicella and herpes-zoster, or shingles. These diseases have as common aetiological agent the varicella-zoster virus (VZV). Zoster can more likely occur in aged people with declining cell-mediated immunity. The general concern is that universal varicella vaccination might lead to more cases of zoster: with more vaccinated children exposure of the general population to varicella infectives become smaller and thus a larger proportion of older people will have weaker immunity to VZV, leading to more cases of reactivation of zoster. Our compartment model shows that only two possible equilibria exist, one without varicella and the other one where varicella and zoster both thrive. Threshold quantities to distinguish these cases are derived. Cost estimates on a possible herd vaccination program are discussed indicating a possible tradeoff choice.
\end{abstract}

Keywords and phrases: varicella zoster virus, vaccine, cost-effectiveness, epidemics

Mathematics Subject Classification: 92D25, 92D30

\section{Introduction}

Varicella (chickenpox) and herpes-zoster (also called shingles) have as common aetiological agent the varicella-zoster virus (VZV). Before a vaccine was developed in 1994, chickenpox was a common contagious childhood disease that produces itchy blisters but rarely caused serious problems. However, if adults who did not have the disease as children contract it, it could cause more serious complications.

Shingles is caused by a reactivation of the virus that causes chickenpox. Once one has had chickenpox, the VZV lies dormant in his/her nerves and can re-emerge as shingles. The transmission of varicella occurs by coughing and sneezing, which are highly contagious means of spreading the virus, by direct contact, and by aerosolization of virus from skin lesions, whereas shingles cannot be passed from one person to another by air. However, the virus that causes shingles, the VZV, can be spread from a person with active shingles to a person who has never had chickenpox. In such cases, the person exposed to the virus might develop chickenpox, but they would not develop shingles. The virus is spread through direct

\footnotetext{
${ }^{*}$ Corresponding author. E-mail: ezio.venturino@unito.it
} 
contact with fluid from the rash blisters, not through sneezing, coughing or casual contact. Shingles, which is characterized by a rash of blisters, can be very painful but is not life-threatening.

Varicella vaccine can prevent this disease. Implementation of the universal varicella vaccination program was followed by a rapid decline in incidence of the disease. The initially recommended one-dose schedule provided only limited protection. Currently then, two doses of vaccine are recommended for children, adolescents, and adults. The interval between the first and the second dose may be short, but it should be at least one month, standard, 3 to 7 years apart, or even longer, depending on varicella epidemiology [4]. Thus, the decision concerning an optimal vaccination schedule depends greatly on the local epidemiological situation [18]. Unfortunately, whatever the schedule, the vaccine-induced immunity wanes. Vaccine efficacy appears to decline with age: from an overall efficacy against herpes-zoster of $64 \%$ when vaccinating at 60-69 years to $38 \%$ among those over 70 years of age [16], [17]. Wild-type VZV infections in those who have been previously vaccinated are called "breakthrough cases". Individuals who become infected with VZV after vaccination have a milder case of varicella with fewer lesions, so that they are generally less infectious than infected individuals who were never vaccinated [19].

The major concern is that universal varicella vaccination might lead to more cases of zoster [10], [24]. Zoster is more likely to occur in people when their cell-mediated immunity declines with age. As the fraction of vaccinated children increases, exposure of the general population to varicella infectives become less frequent. Because fewer people infected in childhood are boosted as they age, a larger proportion of older people will have weaker immunity to VZV, so that zoster may reactivate in more people.

In this paper we use a decision analytical model to assess the effectiveness and cost-effectiveness of routine vaccination against varicella and zoster. The model is formulated in Section 2 and analysed in Section 3. The simulations are presented in Section 4. In Section 5 the model is used to estimate the cost-effectiveness of vaccination against zoster.

\section{Model Formulation}

We consider a total population $N$ which is subdivided into susceptibles individuals, $S$, varicella infectious individuals, $I$, vaccinated individulas, $V$, asymptomatics, $A$ and zoster individuals, $Z$.

A flow diagram is given in Figure 1; and the associated variables and parameters of the model are described in Table 1.

The model is given by the following dynamical system:

$$
\begin{gathered}
\dot{S}=\Pi-\beta S I-\rho S-\mu S \\
\dot{I}=\beta S I+\beta_{1} V I-\gamma I-\mu I \\
\dot{V}=\rho S-\beta_{1} V I-\sigma V-\mu V \\
\dot{A}=\gamma I+\epsilon Z-\delta A-\mu A \\
\dot{Z}=\delta A+\sigma V-\epsilon Z-\mu Z
\end{gathered}
$$

The parameters' meanings are as follows: $\Pi$ denotes the immigration rate into class $S$; $\beta$ is the transmission coefficient of the varicella infection and represents the number of adequate contacts leading to new cases per individual per time unit, i.e., the effective contact rate; $\rho$ is the vaccination rate for a susceptible individual and $\mu$ the natural mortality rate; $\sigma$ represents the progress rate of zoster (activation) in a vaccinated individual and $\delta$ the rate of reactivation of zoster by age in an asymptomatic individual. Finally, both varicella and zoster infectious individuals recover with rate constants $\gamma$ and $\epsilon$ respectively. We define $\beta_{1}=k \beta$, with $0 \leq k \leq 1$, as the transmission coefficient of the disease after vaccination. The parameter $k$ illustrates the effect of immunological memory. Thus it is the factor that reduces the risk of varicella infection. In the case $k=0$, the vaccine is effective and the immunological memory developed against varicella infection does not wane over time; whereas $k=1$ implies that the vaccine is totally useless to induce immunological memory. From now on we make the realistic assumption that 


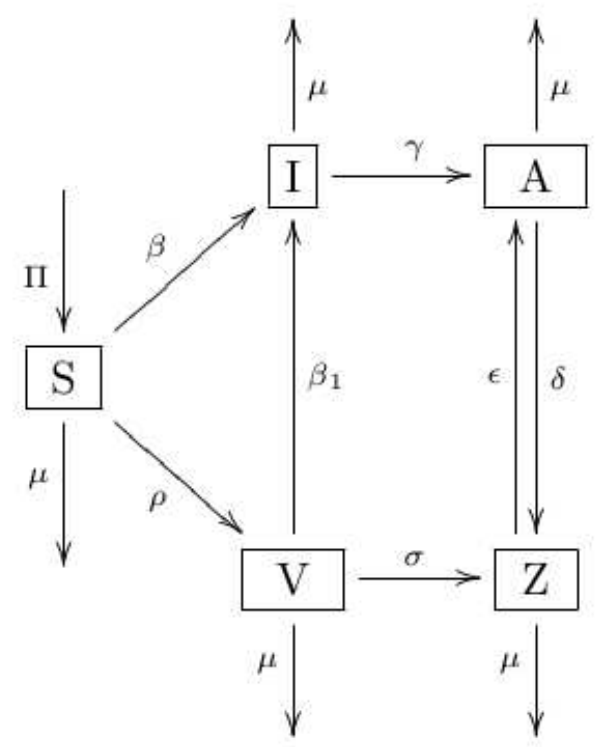

Figure 1. The flow diagram for the model (2.1). Individuals are classified as susceptible $(S)$, infectious $(I)$, vaccinated $(V)$, asymptomatics, $(A)$ and zoster $(Z)$.

vaccination elicit immune response, but fails to offer long-lasting protection against varicella infection, i.e. $0<k<1$.

In the model described by Brisson et al. [3] individuals who had been immunised against varicella were not able to develop zoster. Although zoster from the vaccine-type virus is very unlikely, it can occur, so that a small fraction of those in the vaccinated class $(V)$ could get vaccine type zoster and move into the zoster class $(Z)$. In our model this possibility is allowed for, as studies suggest that vaccinated individuals can, in fact, develop herpes-zoster [12].

Finally it is important to remark that a zoster individual is an infectious who cannot transmit the infection to other individuals by air. The transmission of VZV from people with zoster can occur, but is much less likely than from people with primary varicella [13], [2], [5]. Therefore we assume that zoster individuals are infected, but not infectious individuals, i.e., they are not able to propagate the infection.

We now describe in detail each equation of (2.1). Susceptibles individuals, first equation, are recruited at rate $\Pi$, and leave the class either by acquiring the varicella infection following contact with varicella infectious individuals, or by being vaccinated, or by dying.

Taking into account that the interval between the first- and the second-dose varicella vaccination may be short [18], and in any case the average intervaccination time of two months is short compared to the lifetime of an individual, here we could simply assume the one-dose varicella vaccination schedule. In other words, the one or possibly two doses vaccination will eventually lead to immunization, and the parameter $\rho$ will denote the rate at which the latter is obtained, no matter how in fact the vaccine is administered.

Further, in the second equation we assume that susceptible individuals become varicella infectious individuals via a successful contact between an infectious with a susceptible at rate $\beta$, or a vaccinated one, at lower rate $\beta_{1}$, when the vaccine protection wanes. The varicella-infectious individuals also leave this class by being healed and therefore by migrating into the class of asymptomatics, i.e. those who have been exposed to the virus and will carry it for life, although the latter may or may not be reactivated. This class is also subject to natural mortality. 
TABLE 1. Description of variables and parameters for model (2.1)

\begin{tabular}{ll}
\hline \hline Variables & Description \\
\hline$S$ & susceptible individuals \\
$I$ & varicella-infectious individuals \\
$V$ & vaccinated individuals \\
$A$ & asymptomatic individuals \\
$Z$ & zoster individulas \\
\hline \hline Parameters & Description \\
\hline$\Pi$ & susceptibles recruitment rate \\
$\beta$ & transmission coefficient of the susceptible individuals \\
$\beta_{1}$ & transmission coefficient of the vaccineted individuals \\
$\rho$ & vaccination rate for susceptible individuals \\
$\mu$ & natural mortality rate \\
$\sigma$ & progress rate of zoster - activation \\
$\delta$ & (yearly rate of vaccine-type zoster in vaccinated individuals) \\
& reactivation rate of zoster by age in an asymptomatic individuals \\
$\gamma$ & (yearly rate of zoster per 100 000 people) \\
$\epsilon$ & recovery rate from varicella infection \\
\hline \hline
\end{tabular}

The equation for vaccinated shows that these individuals are recruited at rate $\rho$ from the class of susceptibles, and can migrate into the class of infectious. This can happen in two circumstances. Firstly, if the vaccine protection wanes, at rate $\beta_{1}$. Secondly, assuming that zoster from the vaccine-type virus can occur, here we can also assume that the vaccinated individuals move to the zoster class at rate $\sigma$. Finally, vaccinated individuals are also subject to natural mortality.

Next, the asymptomatics dynamics is described in the fourth equation. They are coming either from the infectious at the disease-recovery rate $\gamma$ or from the zoster individuals at rate $\epsilon$, first two terms, and can leave after virus reactivation, due to a decrease of the immunitary defense system, to become zoster at rate $\delta$, or else via natural mortality, these outcomes being modeled by the last two terms.

Lastly, the zoster individuals enter the class either from the asymptomatic or the vaccinated classes, at respective rates $\delta$ and $\sigma$ and leave it by recovery to the class of asymptomatics at rate $\epsilon$, or by dying.

Finally, since the physical situation being modelled concerns human populations, all the dependent variables and parameters of the model are assumed to be nonnegative.

\section{Model analysis}

From the second equation (2.1) we find

$$
I \cdot\left(\beta S+\beta_{1} V-(\gamma+\mu)\right)=0 .
$$

from which two types of equilibria are seen to exist, the chickepox-free one, in which $I=0$ and the one in which both chickepox and zoster coexist, $I \neq 0$.

We analyze each one of them separately. For stability purposes, we need also the Jacobian $J \equiv$ $J(S, I, V, A, Z)$ of (2.1), namely

$$
\left(\begin{array}{ccccc}
-\rho-\mu-\beta I & -\beta S & 0 & 0 & 0 \\
\beta I & \beta S+\beta_{1} V-\gamma-\mu & \beta_{1} I & 0 & 0 \\
\rho & -\beta_{1} V & -\beta_{1} I-\sigma-\mu & 0 & 0 \\
0 & \gamma & 0 & -\delta-\mu & \epsilon \\
0 & 0 & \sigma & \delta & -\epsilon-\mu
\end{array}\right)
$$




\subsection{Varicella-free equilibrium}

The population levels of this equilibrium $E_{1}=\left(S_{1}, 0, V_{1}, A_{1}, Z_{1}\right)$ are found to be

$$
\begin{aligned}
S_{1} & =\frac{\Pi}{\rho+\mu}, \\
V_{1} & =\frac{\rho \Pi}{(\rho+\mu)(\sigma+\mu)}, \\
A_{1} & =\frac{\epsilon \rho \sigma \Pi}{\mu(\rho+\mu)(\sigma+\mu)(\epsilon+\delta+\mu)}, \\
Z_{1} & =\frac{\Pi \rho \sigma(\delta+\mu)}{\mu(\rho+\mu)(\sigma+\mu)(\epsilon+\delta+\mu)} .
\end{aligned}
$$

This equilibrium is always feasible, since all parameters are nonnegative.

We thus turn to its stability analysis. Substituting the components of $E_{1}$ into (3.1) we find that with $I_{1}=0$ three eigenvalues of $J\left(E_{1}\right)$ are immediately obtained, namely: $-(\rho+\mu),-(\sigma+\mu)$ and

$$
\beta S_{1}+\beta_{1} V_{1}-(\gamma+\mu)=\frac{\beta \Pi(\sigma+\mu)+\rho \Pi \beta_{1}-(\gamma+\mu)(\rho+\mu)(\sigma+\mu)}{(\rho+\mu)(\sigma+\mu)} .
$$

The remaining ones are the roots of the quadratic equation,

$$
\lambda^{2}+(\delta+\epsilon+2 \mu) \lambda+\mu(\delta+\epsilon+\mu)=0 .
$$

which explicitly are found to be $-\mu,-(\delta+\epsilon+\mu)$.

Hence, there is only one possibly nonnegative eigenvalue given by (3.3), on which stability depends. In this way, the equilibrium point $E_{1}$ is stable if only if the following condition holds,

$$
\beta \Pi(\sigma+\mu+k \rho)-(\gamma+\mu)(\rho+\mu)(\sigma+\mu)<0 .
$$

Letting

$$
\beta_{s}=\frac{(\gamma+\mu)(\rho+\mu)(\sigma+\mu)}{\Pi(\sigma+\mu+k \rho)}
$$

we can rewrite (3.4) as

$$
\frac{\beta}{\beta_{s}}<1
$$

One way to deal with this situation consists in introducing the concept of reproduction number in presence of vaccine $R_{v a c c}$, [7], so that the stability of the equilibrium point $E_{1}$ holds for

$$
R_{\text {vacc }}=\frac{\beta}{\beta_{s}}<1
$$

We can also calculate the basic reproduction number. Setting $\rho=0$ into (2.1), $E_{1}$ simplifies to

$$
S_{1 \mid \rho=0}=\frac{\Pi}{\mu}, \quad I_{1 \mid \rho=0}=0, \quad V_{1 \mid \rho=0}=0, \quad A_{1 \mid \rho=0}=0, \quad Z_{1 \mid \rho=0}=0,
$$

and the simplified eigenvalues are the double one $-\mu$ and

$$
\frac{\beta \Pi-\mu(\gamma+\mu)}{\mu}, \quad-(\sigma+\mu), \quad-(\delta+\epsilon+\mu) .
$$

Letting

$$
\beta_{L}=\frac{\mu(\gamma+\mu)}{\Pi}
$$


stability is then regulated by the inequality $\beta<\beta_{L}$, or

$$
R_{0}<1
$$

having defined the basic reproduction number as

$$
R_{0}=\frac{\beta}{\beta_{L}} .
$$

Further, $R_{\text {vacc }}$ can now be rewritten in terms of $R_{0}$ as follows

$$
R_{v a c c}=R_{0} \frac{\mu(k \rho+\sigma+\mu)}{(\rho+\mu)(\sigma+\mu)}
$$

from which $R_{0}>R_{v a c c}$ follows, since

$$
\frac{\mu(k \rho+\sigma+\mu)}{(\rho+\mu)(\sigma+\mu)}<1
$$

is equivalent to

$$
k \mu \rho<\rho \sigma+\rho \mu
$$

from which dividing by $\rho$ we get $k \mu<\sigma+\mu$ i.e.

$$
\sigma+\mu \cdot(1-k)>0
$$

which is verified since $0 \leq k \leq 1$.

The stability analysis can also be recast into other forms. In terms of $\rho$, starting from (3.4) by rewriting it we find

$$
\rho>\frac{\beta \Pi(\sigma+\mu)-\mu(\gamma+\mu)(\sigma+\mu)}{(\gamma+\mu)(\sigma+\mu)-k \Pi \beta} .
$$

If the right hand side is negative, evidently any positive amount of vaccination will be enough to stabilize the equilibrium so that varicella is eradicated. The right hand side is positive if one of the two conditions hold:

$$
\frac{\mu(\gamma+\mu)}{\Pi}<\beta<\frac{(\gamma+\mu)(\sigma+\mu)}{k \Pi} ; \quad \frac{\mu(\gamma+\mu)}{\Pi}>\beta>\frac{(\gamma+\mu)(\sigma+\mu)}{k \Pi} .
$$

If either one of (3.13) holds, then to eradicate the disease one needs a rate of vaccination which exceeds the following value

$$
\rho_{s}=\frac{\beta \Pi(\sigma+\mu)-\mu(\gamma+\mu)(\sigma+\mu)}{(\gamma+\mu)(\sigma+\mu)-k \Pi \beta} .
$$

Further, note that (3.4) can also be recast in the following form

$$
\rho\left[\frac{(\gamma+\mu)(\sigma+\mu)}{\mu(\gamma+\mu)}-\frac{k \Pi \beta}{\mu(\gamma+\mu)}\right]>\frac{\beta \Pi(\sigma+\mu)}{\mu(\gamma+\mu)}-\frac{\mu(\gamma+\mu)(\sigma+\mu)}{\mu(\gamma+\mu)},
$$

from which, recalling (3.10) and (3.8), we have

$$
\rho>\frac{\mu(\sigma+\mu)\left(R_{0}-1\right)}{(\sigma+\mu)-k \mu R_{0}} .
$$

Again if the right had side is negative, any positive vaccination rate ensures the eradication of the disease, here. On the other hand, the right hand side is positive for one of the alternative conditions

$$
1<R_{0}<\frac{\sigma+\mu}{k \mu} ; \quad 1>R_{0}>\frac{\sigma+\mu}{k \mu} .
$$

These once again ensure that $\rho_{s} \geq 0$, where this threshold quantity can also be written as

$$
\rho_{s}=\frac{\mu(\sigma+\mu)\left(R_{0}-1\right)}{(\sigma+\mu)-k \mu R_{0}}
$$

and the varicella-free equilibrium is attained if the vaccination rate exceeds $\rho_{s}$. 


\subsection{Coexistence Equilibrium}

Solving the nonlinear system $(2.1)$, for $I \neq 0$, we find the following population values for the coexistence equilibrium $E_{2}=\left(S_{2}, I_{2}, V_{2}, A_{2}, Z_{2}\right)$

$$
\begin{aligned}
S_{2} & =\frac{\Pi}{\beta I_{2}+\rho+\mu}, \\
V_{2} & =\frac{\rho \Pi}{\left(\beta I_{2}+\rho+\mu\right)\left(\beta_{1} I_{2}+\sigma+\mu\right)}, \\
A_{2} & =\frac{\delta \gamma I_{2}(\epsilon+\mu)\left(\beta I_{2}+\rho+\mu\right)\left(\beta_{1} I_{2}+\sigma+\mu\right)+\epsilon \rho \sigma \Pi}{\mu\left(\beta I_{2}+\rho+\mu\right)\left(\beta_{1} I_{2}+\sigma+\mu\right)(\delta+\epsilon+\mu)}, \\
Z_{2} & =\frac{\delta \gamma I_{2}\left(\beta I_{2}+\rho+\mu\right)\left(\beta_{1} I_{2}+\sigma+\mu\right)+\rho \sigma \Pi(\delta+\mu)}{\mu\left(\beta I_{2}+\rho+\mu\right)\left(\beta_{1} I_{2}+\sigma+\mu\right)(\delta+\epsilon+\mu)},
\end{aligned}
$$

where the value of $I_{2}$ is determined by the roots of the following quadratic

$$
\begin{aligned}
& a I^{2}+b I+c=0, \\
a= & k \beta^{2}(\gamma+\mu)>0, \\
b= & (\gamma+\mu)[k \beta(\rho+\mu)+\beta(\sigma+\mu)]-k \beta^{2} \Pi, \\
c= & (\gamma+\mu)(\rho+\mu)(\sigma+\mu)-\Pi[k \beta \rho+\beta(\sigma+\mu))] .
\end{aligned}
$$

All populations in (3.17) are nonnegative, so feasibility is obtained by discussing the sign of the roots of (3.18). Descartes' rule ensures one positive solution for $c<0$, independently of the sign of $b$, and two positive ones for $b<0$ and $c>0$. Now, $b>0$ is equivalent to

$$
\beta<\frac{(\gamma+\mu)(k(\rho+\mu)+\sigma+\mu)}{k \Pi} \equiv \beta_{b}
$$

and $c>0$ can be rewritten as

$$
\beta<\frac{(\gamma+\mu)(\rho+\mu)(\sigma+\mu)}{\Pi(k \rho+\sigma+\mu)} \equiv \beta_{c} .
$$

It is easily verified that $\beta_{b}>\beta_{c}$. Therefore for $\beta<\beta_{c}$ there are only negative roots, while for $\beta>\beta_{c}$ there is one positive root. Now $\beta_{c}$ recalls the feasibility condition for the varicella-free equilibrium (3.5), (3.6). The feasibility condition for the coexistence equilibrium is then

$$
\beta>\frac{(\gamma+\mu)(\rho+\mu)(\sigma+\mu)}{\Pi(k \rho+\sigma+\mu)},
$$

recalling again (3.5), so that it can be rewritten as $\beta>\beta_{s}$, from which finally

$$
1<\frac{\beta}{\beta_{s}} \equiv R_{v a c c}
$$

This result is the opposite of the stability condition for the varicella-free equilibrium (3.9). When the disease-free equilibrium is stable, the coexistence one is not feasible. Vice versa, when there is coexistence, the disease-free equilibrium must be unstable. Further, for $I_{2}=0$, the coexistence equilibrium becomes the varicella-free one, compare their respective components (3.18) and (3.2). Thus mathematically we have here a transcritical bifurcation: at $R_{v a c c}=1$ equilibrium $E_{1}$ concides with $E_{2}$ and when $R_{v a c c}$ grows larger, the former loses its stability in favor of the latter.

Note that the coefficient of the polynomial (3.18) can be rewritten as

$$
c=(\gamma+\mu)(\rho+\mu)(\sigma+\mu)\left(1-R_{\text {vacc }}\right) .
$$


Therefore, for $R_{v a c c}>1, c<0$, there is only one positive root for the quadratic (3.18), i.e., the coexistence equilibrium is feasible and must be stable. For $R_{v a c c}<1, c>0$, there are two negative solutions for the polynomial (3.18), i.e. only the varicella-free equilibrium exists and it is stable.

We now elaborate this result in terms of the vaccination rate $\rho$ as done in the previous case. Expressing (3.21) in terms of $\rho$ we find

$$
\rho<\frac{\mu(\gamma+\mu)(\sigma+\mu)-\beta \Pi(\sigma+\mu)}{k \beta \Pi-(\gamma+\mu)(\sigma+\mu)} \equiv \rho_{s} .
$$

If the right hand side of (3.23) is negative then (3.21) does not hold, and the coexistence equilibrium is then infeasible. For (3.21) to hold, we need a positive right hand side of (3.23), which is ensured by

$$
\rho<\frac{\mu(\sigma+\mu)\left(R_{0}-1\right)}{(\sigma+\mu)-k \mu R_{0}} .
$$

In order that this inequality be satisfied, the right hand side must be positive. Then we obtain that the conditions for which the coexistence equilibrium is feasible coincide with the stability conditions (3.15) for the chickepox-free equilibrium earlier found.

The stability analysis of the interior coexistence equilibrium hinges on the full Jacobian (3.1). Luckily the characteristic equation of this matrix factors, to give the following two explicit eigenvalues as roots of a quadratic,

$$
\lambda_{1}=-\mu, \quad \lambda_{2}=-(\delta+\epsilon+\mu),
$$

which are both negative and therefore do not influence the stability issue. The remaining ones the are roots of the monic cubic

$$
\lambda^{3}+a_{2} \lambda^{2}+a_{1} \lambda+a_{0}=0
$$

with

$$
\begin{aligned}
a_{2}= & \left(\beta-\beta_{1}\right) I_{2}+\beta S_{2}+\beta_{1} V_{2}+\rho-\gamma-\mu-\sigma, \\
a_{1}= & \left(-\rho-\mu-\beta I_{2}\right)\left(\beta S_{2}+\beta_{1} V_{2}-\beta_{1} I_{2}-\gamma-\sigma-2 \mu\right)+ \\
& \left(\beta S_{2}+\beta_{1} V_{2}-\gamma-\mu\right)\left(-\beta_{1} I_{2}-\sigma-\mu\right)-\beta_{1}^{2} I_{2} V_{2}, \\
a_{0}= & \left(\rho+\mu+\beta I_{2}\right)\left[\left(\beta S_{2}+\beta_{1} V_{2}-\gamma-\mu\right)\left(-\beta_{1} I_{2}-\sigma-\mu\right)-\beta_{1}^{2} I_{2} V_{2}\right]+ \\
& \beta S_{2}\left(\beta \beta_{1} I_{2}^{2}+\beta_{1} \rho I_{2}\right) .
\end{aligned}
$$

To study stability using the Routh-Hurwitz conditions, since the signs of all coefficients are not easily determined, is quite involved. Therefore we will investigate the stability only via numerical methods.

\section{Simulations}

The first results show that coexistence can indeed been achieved, as the theoretical analysis of the stability of this equilibrium is missing, Figure 2.

Only for simulations purposes, we have chosen the fixed parameters as follows. We select $k=0.02$ assuming the vaccine to be $98 \%$ effective; $\rho=0.85$ assuming to be able to vaccinate $85 \%$ of the susceptibles; $\gamma=52.142$ years $^{-1}$, by converting into the time unit, the year, the disease infectious period, which is 7 days; $\delta$ and $\sigma$ are the rates at which zoster can appear, respectively after varicella and after the vaccine administration; these parameters are estimated to be 70 and 17 persons every 100.000 per year; $\epsilon=18.25$ years $^{-1}$ represents the reciprocal of the healing time, which is assumed to be 20 days; $\mu=1 / 85$ years $^{-1}$ is the reciprocal of life expectancy; assuming the average lifetime to be 85 years; the immigration rate is taken $\Pi=100.000$ people years $^{-1}$, since the rate of progress of zoster is expressed in terms of this population unit. 
From these values, we find

$$
\beta_{s}=\frac{(\gamma+\mu)(\rho+\mu)(\sigma+\mu)}{\Pi(k \rho+\sigma+\mu)}=9.27133 \cdot 10^{-5} .
$$

and thus we take values of $\beta$ just above and below $\beta_{s}$, namely $\beta=9 \cdot 10^{-4}$ people $^{-1}$ year $^{-1}$ and $\beta=9 \cdot 10^{-5}$ people $^{-1}$ year $^{-1}$.

In Figure 2 , for $\beta>\beta_{s}$, the system settles to the coexistence equilibrium $E_{2}$ and for $\beta<\beta_{s}$, the system settles to the varicella-free equilibrium $E_{1}$. These equilibria are explicitly given by

$$
E_{1}=(116041,0,8264536,119341,82), \quad E_{2}=(23829,3705,257599,8214548,317),
$$

when $\beta<\beta_{s}$.
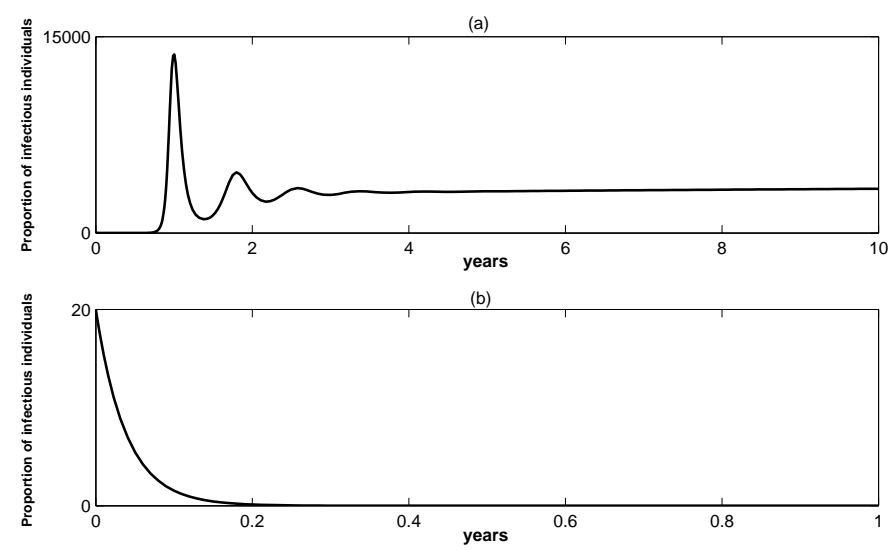

FIgURE 2. Profiles of populations for infectious individuals where (a) $\beta>\beta_{s}$ : coexistence equilibrium; (b) $\beta<\beta_{s}$ : varicella-free equilibrium. System's behavior for (a) $\beta=9 \cdot 10^{-4}$ and (b) $\beta=9 \cdot 10^{-5}$.

\subsection{System's behavior as as function of $\beta$}

We now perform further numerical experiments to investigate the behavior of the coexistence equilibrium when the parameters change. First we used as bifurcation parameter $\beta$. In a second phase, we included also the vaccination rate $\rho$. Finally, together with these two parameters, also the influence of the immmigration rate $\Pi$ has been considered.

In Figure 3 we have the bifurcation diagram of $I$ as a function of the disease incidence $\beta$. There is a transcritical bifurcation (forward bifurcation) at $\beta=\beta_{s}=9.27133 \cdot 10^{-5},(3.5)$ or $R_{\text {vacc }}=1$. At that point $E_{1}$ becomes unstable, while $E_{2}$ instead becomes feasible and stable. The transcritical bifurcation affects also the other populations. A forward bifurcation occurs, that is, for $R_{v a c c}<1$, the varicella-free equilibrium is locally asymptotically stable, and for $R_{v a c c}>1$, the coexistence equilibrium is locally asymptotically stable. The diagram of the forward bifurcation corresponding to equation (3.18) as a function of $R_{\text {vacc }}$ is depicted in Figure 2 .

The equilibrium change entails lower population values in the classes of susceptibles and vaccinated, while the populations of asymptomatic individuals and of zoster increase as the infected do. The reason is that $S$ decreases for larger values of infected, since they become varicella infectious individuals. Therefore also the number of susceptibles that can be vaccinated decreases. A larger value of $I$ entails that more asymptomatics and thus also more zoster individuals will appear in the population. 

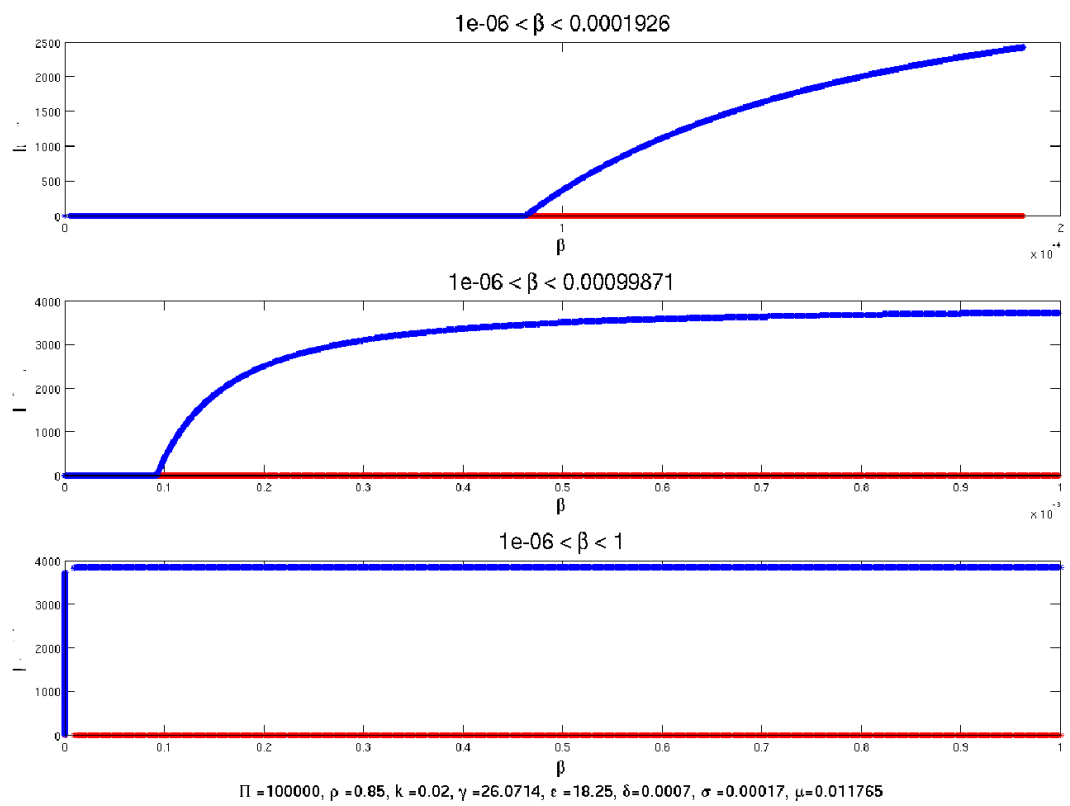

FiguRE 3. Bifurcation diagram of $I$ as function of $\beta$

\subsection{System's behavior as as function of $\beta$ and $\rho$}

We now investigate the modifications in the equilibria when both disease incidence and vaccination rate change. In this case we therefore give a bifurcation diagram of $I$ as function of $\beta$ and $\rho$, Figure 4 .

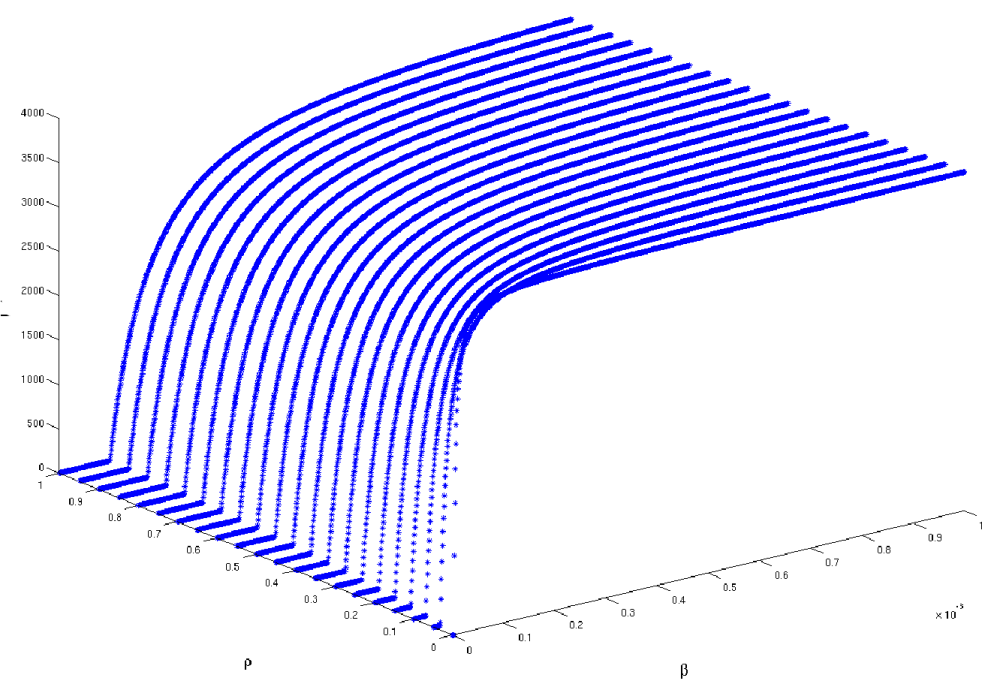

Figure 4. Bifurcation diagram of $I$ as function of $\beta$ and $0 \leq \rho \leq 1$ 
Note that the curve intersection of the surface with the $\beta-\rho$ plane gives the locus of all the threshold points $\beta_{s}$, (3.5) which vary between the extreme values given by

$$
\beta_{s \mid \rho=0}=3.06861 \cdot 10^{-6}, \quad \beta_{s \mid \rho=1}=9.86255 \cdot 10^{-5}
$$

In fact from (3.5) we have that $\beta_{s} \equiv \beta_{s}(\rho)$, and Figure 4 depicts graphically this dependence.

\subsection{Threshold behavior in terms of $\rho$ and $\Pi$}

Here we consider the threshold $\beta_{s}$ behavior in terms of the vaccination rate $\rho$ and the immigration rate $\Pi$, namely $\beta_{s}=\beta_{s}(\rho, \Pi)$, see once again (3.5). We take as domain of the parameter plane the cartesian product of the intervals $\rho \in[0,1]$ and $\Pi \in[0,10000000]$.

In Figure 5 for large values of $\Pi$, say $10^{7}$, the threshold $\beta_{s}$ tends the faster to zero, the larger $\rho$ is and conversely, when $\Pi$ approaches 0 the threshold grows the faster very large, the closer to 1 is the vaccination rate. The minimal value we have obtained in the simulations is $\beta_{s}=3.06861 \cdot 10^{-8}$. Thus the smaller the immigration rate, the smaller the chances are of eradicating the disease.

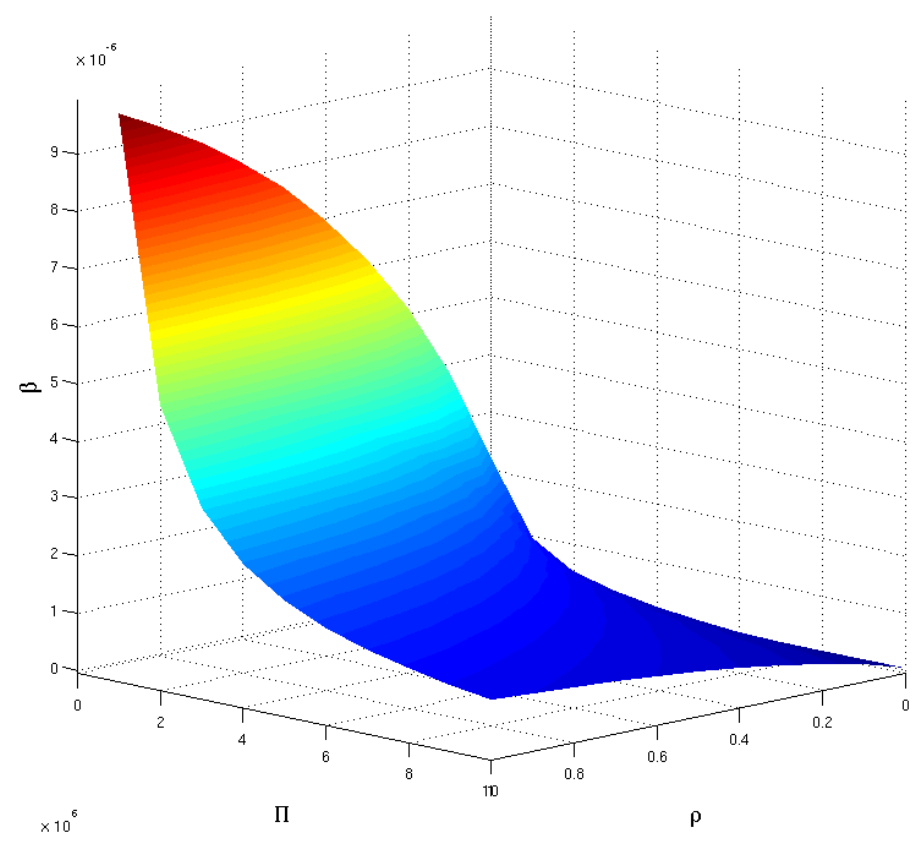

Figure 5. Plot of $\beta$ as a function of $\rho$ and $\Pi$

\section{Cost estimates}

Here we analyze the costs that are incurred for treatment and other issues like doctors' bills, laboratory tests, hospitalizations, workdays or schooldays lost and so on, see for instance [21]. The data used here are the ones reported in [20]. 
The cost function is obtained as sum of the individuals costs, $c_{i}$ for infected, $c_{v}$ for vaccinated and $c_{z}$ for zoster individuals, over a suitable time span $t_{f}$,

$$
C=\int_{0}^{t_{f}}\left(c_{i} I+c_{v} V+c_{z} Z\right) \mathrm{d} t .
$$

In general this function depends not only on these quantities, but on the disease incidence and the vaccination rate as well, so that

$$
C=C(\beta, \rho, I(t), V(t), Z(t)),
$$

which needs to be minimized. This task has been performed numerically, in view of the complexity of the problem, to get some insight into it.

Using the parameters already appearing in the simulations, we simulate the cost as function of the remaining parameters, in particular at first the disease incidence $\beta$, taking into account also [14,20].

\subsection{Cost as a function of $\beta$}

Let us assume the individual costs to be $306 €, 100 €$ and $200 €$ respectively for infected, vaccinated and zoster individuals. For a population of 500000 individuals, we obtain the value of the average individual cost as

$$
\frac{153 \cdot 10^{6} €}{5 \cdot 10^{5}}=306 €
$$

After obtaining the coexistence equilibrium values, the costs of each subpopulation are evaluated, $C_{I}(t)=c_{i} I(t), C_{V}(t)=c_{v} V(t), C_{Z}(t)=c_{z} Z(t)$ and then the total one $C(t)=C_{I}(t)+C_{V}(t)+C_{Z}(t)$. Repeating the calculation for each value of $\beta$, we thus find the graph of Figure 6 . On summing each

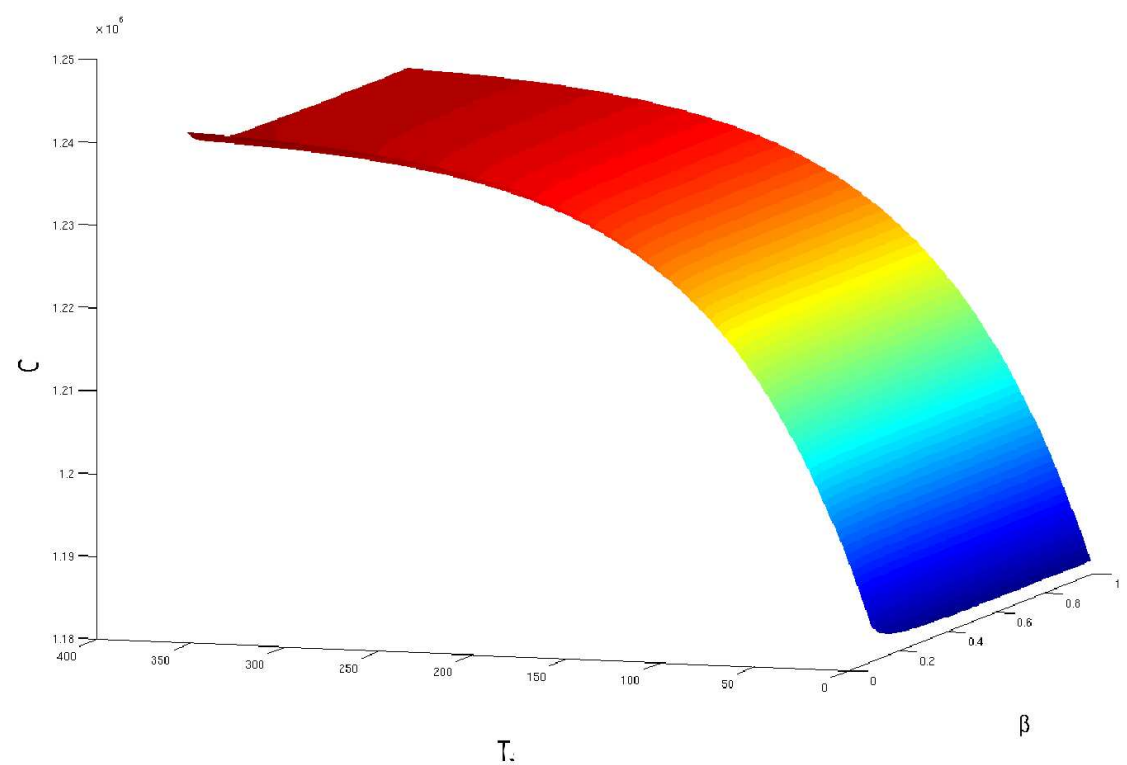

Figure $6 . C$ as function of $\beta$ and time.

instantaneous cost over time and repeatig for each value of $\beta$ we obtain the total cost in time

$$
C_{T} \equiv C_{T}(\beta)=\sum_{t=0}^{365} C(t)
$$


From this, the extremal values are found respectively for $\beta=1$ and $\beta=0$

$$
C_{\min }=4.47181595378536 \cdot 10^{8} €, \quad C_{\max }=2.40930560139391 \cdot 10^{11} € .
$$

There is thus a higher cost with varicella and vaccination, instead of the one with high infectivity. This can be explained by looking at how each subpopulation behaves in time.

Starting from $\beta=0$ we find that the sum of each subpopulation is

$$
\sum I(t)=1, \quad \sum V(t)=2.409258812 \cdot 10^{9}, \quad \sum Z(t)=2.339296341 \cdot 10^{4} .
$$

The largest population is the one of vaccinated individuals, therefore this one will bear the largest weight in the cost calculation, in spite of having its pro-capita cost smaller than the other subpopulations. Infected remain constant at 1 , since the disease does not spread. Zoster individuals come only from vaccine failures. The costs for each class are

$$
C_{I}=306, €, \quad C_{V}=2.40925881241 \cdot 10^{11} €, \quad C_{Z}=4.67859268124 \cdot 10^{6} € .
$$

On summing we find $C_{T}=2.40930560139391 \cdot 10^{11} €$.

We now analyze the case $\beta=1$. The sum in each class is now

$$
\sum I=1.39936520 \cdot 10^{6}, \quad \sum V=105.497125, \quad \sum Z=9.48264717 \cdot 10^{4} .
$$

Here the largest class is $I$, containing about $10^{6}$ individuals, while formerly the peak was at $10^{11}$. For the costs we have

$$
C_{I}=4.282057513 \cdot 10^{8} €, \quad C_{V}=1.054971 \cdot 10^{4} €, \quad C_{Z}=1.896529435 \cdot 10^{7} €
$$

from which the total cost $C_{T}=4.471815954 \cdot 10^{8} €$.

We then analyze two intermediate situations for $\beta$, respectively above and below the threshold $\beta_{s}=$ $3.06861 \cdot 10^{-8},(3.5)$, considering the interval $[0,0.00001]$. Repeating the simulation, we obtain Figure 7. The total cost is almost constant, apart from some peaks, at about the level $2.4086 \cdot 10^{11}$, and the maximum is obtained for $\beta=0$. For values of $\beta$ larger than $\beta_{s}$, we consider the interval $[0.0001,1]$, Figure

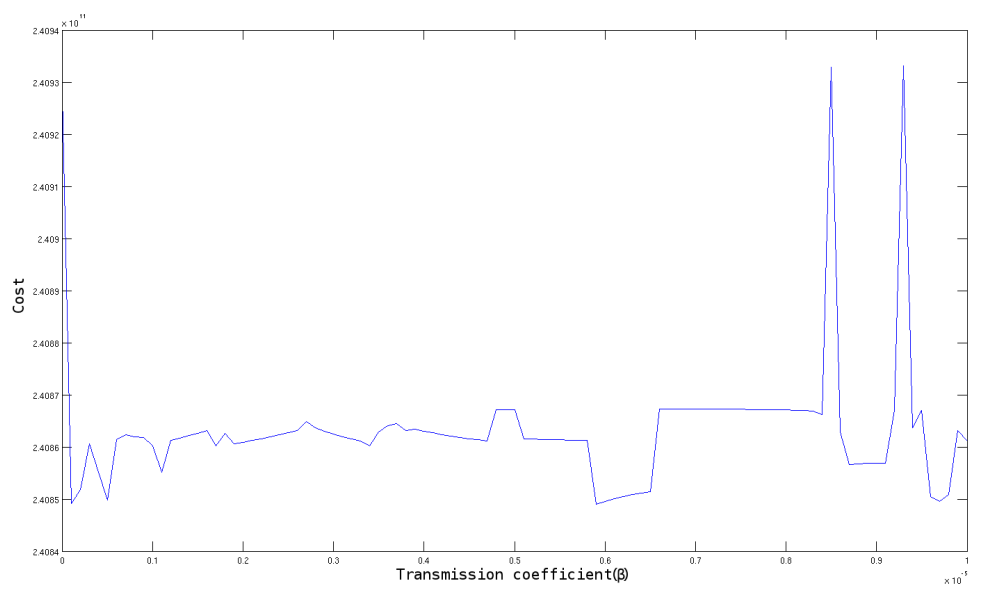

Figure 7. Graph of $C_{T}$ as function of $\beta$ with $\beta \in[0,0.00001]$

8. The values obtained fall in the interval $\left[4.46 \cdot 10^{8}, 4.47 \cdot 10^{8}\right]$. Therefore a vaccination program which aims at involving $85 \%$ of the population is cheaper if the disease incidence has a high value, and it is not worth the case to implement it when $\beta$ falls below the threshold $\beta_{s}$. 


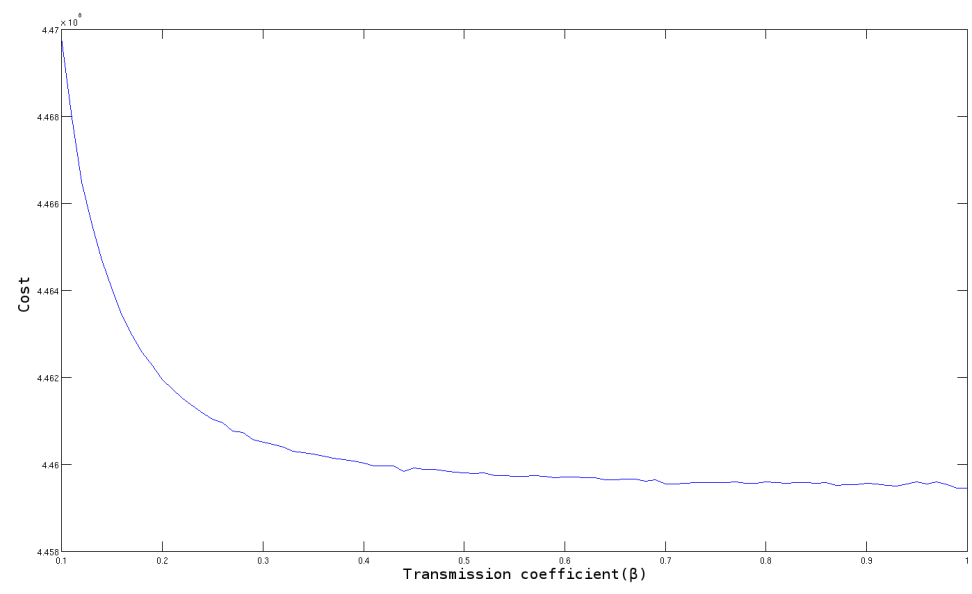

Figure 8. Graph of $C_{T}$ as function of $\beta$ with $\beta \in[0.0001,1]$

\subsection{Cost as a function of $\beta$ and $\rho$}

We now vary both disease incidence and vaccination rate. The intervals in which these are allowed to vary are

$$
\rho \in[0,1], \quad \beta \in[0,0.00001] \cup[0.1,1]
$$

The maximum for $\beta<\beta_{s}$ is

$$
C_{\max }=2.41567255166791 \cdot 10^{11} €
$$

and Figure 9 contains the graphical display of the results. It shows that cost increases with $\rho$ independently of $\beta$, with a maximum at $(\beta, \rho)=(0.000001,1)$. Thus for $\beta<\beta_{s}$ we are better off minimizing the vaccination rate. For $\beta>\beta_{s}$, excluding the trivial case of the origin, with no disease evolution in the

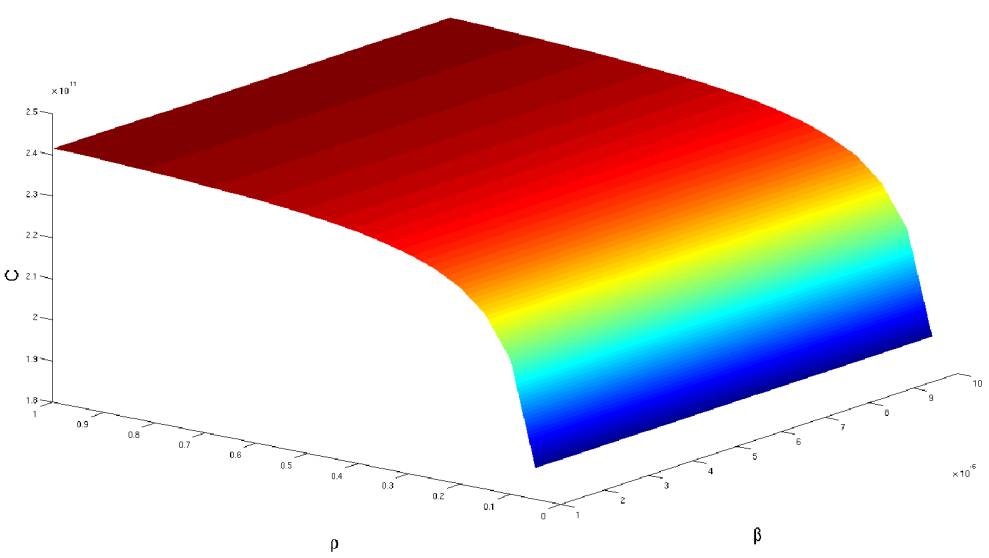

Figure 9. Plot of the total cost as a function of $\beta$ and $\rho$, with $\beta<\beta_{s}$

population, namely $(\beta, \rho)=(0,0)$ with a cost of $306 €$, the minimal cost is, see Figure 10,

$$
C_{\min }=4.471700787594944 \cdot 10^{8} € .
$$


In this case, as $\beta$ decreases and $\rho$ increases, the cost becomes higher, up to the peak $C_{\text {max }_{\text {rel }}}=$ $4.48398374297879 \cdot 10^{8} €$ at $(\beta, \rho)=(0.1,1)$. As $\beta$ grows, the total cost decreases with a minimum

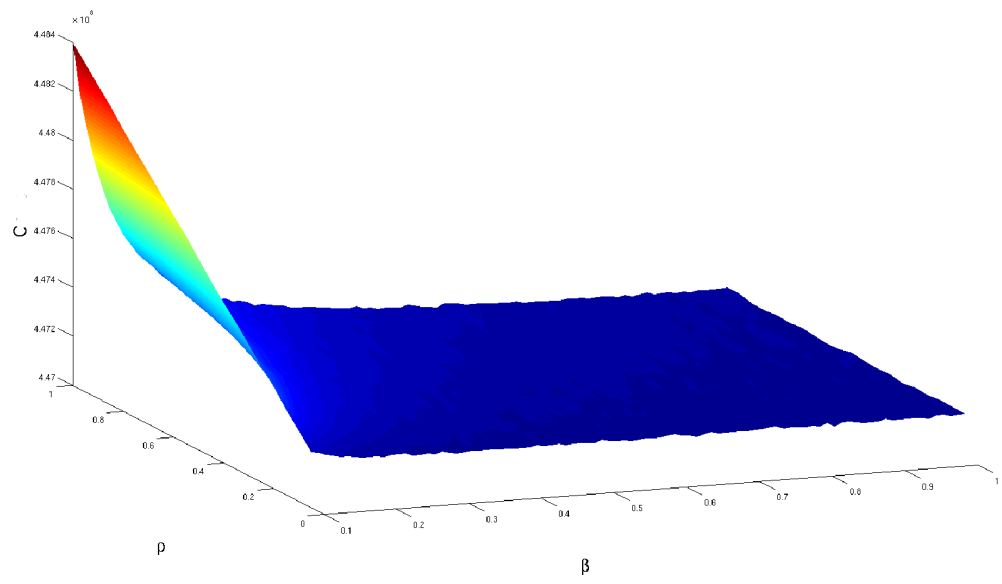

Figure 10. Plot of the total cost as a function of $\beta$ and $\rho$, with $\beta>\beta_{s}$

at $(\beta, \rho)=(1,1)$. But in this case almost all values are between $4.4717 \cdot 10^{8}$ and $4.4723 \cdot 10^{8}$. For smaller $\beta$ and the same $\rho$ the cost is higher.

In summary, a herd vaccination program would make sense if the disease incidence $\beta$ is above the threshold, or if $R_{v a c c}>1$. In these cases costs can be minimized. But the disadvantage is that the disease will not be eradicated, it will remain at an endemic level. Conversely, it is possible to eradicate varicella, but this can be obtained only at large costs. Perhaps the tradeoff choice consists of keeping around the threshold value $\beta_{s}$, since this entails high cost, but not a maximal one together with the possibility of eradicating the disease.

Acknowledgements. S. M. R. gratefully acknowledges the partial support of the progetto Lagrange of the Fondazione ISI, Villa Gualino, viale Settimio Severo 65, 10133 Torino, Italia, within which this research was started. Work performed with the support of Fondazione CRT/Progetto Lagrange.

\section{References}

[1] A. M. Arvin, A. A. Gershon. Live attenuated varicella vaccine. Ann. Rev. Microbiol., 50 (1996), 59-100.

[2] A. M. Arvin, A. A. Gershon. Live attenuated varicella vaccine. Ann Rev Microbiol., 50 (1996), 59-100.

[3] M. Brisson, W. J. Edmunds, N. J. Gay, B. Law, G. De Serres. Modelling the Impact of Immunization on the Epidemiology of Varicella Zoster Virus. Epidemiology and Infection, 125 (2000), 651-669.

[4] CDC. Prevention of varicella: recommendations of the Advisory Committee on Immunization Practices (ACIP). MMWR, 56 (2007), No. RR4, OpenURL.

[5] Centers for Disease Control and Prevention. Prevention of varicella: recommendations of the Advisory Committee on Immunization Practices (ACIP). MMWR, 45 (1997), No. RR-11, 35-40.

[6] S. Deguen, A. Flahault. Impact on Immunization of Seasonal Cycle of Chickenpox. European Journal of Epidemiology, 16 (2000), 1177-1181.

[7] O. Diekmann, J. A. P. Heesterbeek, J. A. J. Metz. On the definition and the computation of the basic reproduction ratio $r_{0}$ in models for infectious diseases in heterogeneous populations. Journal of Mathematical Biology, 28 (1990), No. 4, 365-382, Doi: 10.1007/BF00178324.

[8] W. J. Edmunds, M. Brisson. The effect of vaccination on the epidemiology of varicella zoster virus. Journal of Infection, 44 (2002), 211-219.

[9] J. E. Forde, B. Meeker. A model of varicella-zoster reactivation. Mathematical Biosciences and Engineering, 7 (2010), 765-777. 
[10] G. P. Garnett, N. M. Ferguson. Predicting the effect of varicella vaccine on subsequent cases of zoster and varicella. Rev. Med. Virol., 6 (1996), 151-161.

[11] G. P. Garnett, B. T. Grenfell. The Epidemiology of Varicella-Zoster Virus Infections: The Influence of Varicella on the Prevalence of Herpes Zoster. Epidemiology and Infection, 108 (1992), 513-528.

[12] S. Hambleton, S. P. Steinberg, P. S. La Russa, E. D. Shapiro, A. A. Gershon. Risk of Herpes Zoster in adults immunized with varicella vaccine. J. Infect. Dis., 197 (2008), S196-199.

[13] R. E. Hope-Simpson. The nature of herpes zoster: a long term study and a new hypothesis. Proc. Roy. Soc. Med., 58 (1965), 9-20.

[14] J. Hornberger, K. Robertus. Cost-Effectiveness of a Vaccine To Prevent Herpes Zoster and Postherpetic Neuralgia in Older Adults. Ann Intern Med., 145 (2006), 317-325.

[15] M. Karhunen, T. Leino, H. Salo, I. Davidkin, T. Kilpi, K. Auranen. Modelling the impact of varicella vaccination on varicella and zoster. Epidemiol Infect., 138 (2010), No. 4, 469-481.

[16] M. N. Oxman, M. J. Levin, G. R. Johnson, K. E. Schmader, S. E. Straus, L. D. Gelb, R. D. Arbeit, M. S. Simberkoff, A. A. Gershon, L. E. Davis, A. Weinberg, K. D. Boardman, H. M. Williams, J. Hongyuan Zhang, P. N. Peduzzi, C. E. Beisel, V. A. Morrison, J. C. Guatelli, P. A. Brooks, C. A. Kauffman, C. T. Pachucki, K. M. Neuzil, R. F. Betts, P. F. Wright, M. R. Griffin, P. Brunell, N. E. Soto, A. R. Marques, S. K. Keay, R. P. Goodman, D. J. Cotton, J. W. Gnann, Jr., J. Loutit, M. Holodniy, W. A. Keitel, G. E. Crawford, S.-S. Yeh, Z. Lobo, J. F. Toney, R. N. Greenberg, P. M. Keller, R. Harbecke, A. R. Hayward, M. R. Irwin, T. C. Kyriakides, C. Y. Chan, I. S. F. Chan, W. W. B. Wang, P. W. Annunziato, J. L. Silber. A vaccine to prevent herpes zoster and postherpetic neuralgia in older adults. N. Engl. J. Med., 352 (2005), No. 22, 2271-2284.

[17] M. N. Oxman, M.J. Levin. Vaccination against herpes zoster and postherpetic neuralgia. J Infect Dis., 197 (2008), Suppl. 2, S228-5236.

[18] M. Socan, N. Berginc, J. Lajovic. Varicella susceptibility and transmission dynamics in Slovenia. BMC Public Health, 10:360 (2010), 1-6.

[19] M. C. Schuette, H. W. Hethcote. Modeling the effects of varicella vaccination programs on the incidence of chickenpox and shingles. Bull Math Biol., 61 (1999), No. 6, 1031-1064.

[20] G. Soldato. Nuove strategie preventive vaccinali: vantaggi e limiti del vaccino antivaricella (New preventive vaccine strategies: advantages and limits of the vaccine against chickenpox). Thesis (Advisor Prof. C. Granchelli), University "G. D'Annunzio" of Chieti-Pescara, Italy, 2005.

[21] N. Thiry, P. Beutels, F. Tancredi, L. Romanò, A. Zanetti, P. Bonanni, G. Gabutti, P. Van Damme. An economic evaluation of varicella vaccination in italian adolescents. Vaccine 22, (2004), No. 27-28, 3546-3562.

[22] A. J. van Hoek, N. Gay, W. Opstelten, W. J. Edmunds. Estimating the cost-effectiveness of vaccination against Herpes Zoster in England and Wales. Vaccine, 27 (2009), No. 9, 1454-1467.

[23] A. J. Van Hoek, A. Melegaro, E. W. Zaghenid, J. Edmunds, N. Gay. Modelling the impact of a combined varicella and zoster vaccination programme on the epidemiology of varicella zoster virus in England. Vaccine, 29 (2011), 2411-2420.

[24] M. Wharton. The epidemiology of varicella-zoster virus infections. Infect Dis Clinics N Am, 10 (1996), 571-581. 